\title{
Théologiques
}

\section{Mémoire collective et pardon collectif}

\section{Jean Monbourquette}

Volume 2, numéro 2, octobre 1994

L'esprit

URI : https://id.erudit.org/iderudit/602409ar

DOI : https://doi.org/10.7202/602409ar

Aller au sommaire du numéro

Éditeur(s)

Faculté de théologie de l'Université de Montréal

ISSN

1188-7109 (imprimé)

1492-1413 (numérique)

Découvrir la revue

Citer cet article

Monbourquette, J. (1994). Mémoire collective et pardon collectif. Théologiques, 2(2), 91-116. https://doi.org/10.7202/602409ar d'utilisation que vous pouvez consulter en ligne.

https://apropos.erudit.org/fr/usagers/politique-dutilisation/ 
Théologiques 2/2 (1994) 91.116.

\title{
Mémoire collective et pardon collectif
}

\author{
Jean MONBOURQUETTE \\ Institut de pastorale \\ Université Saint-Paul
}

\section{Introduction}

L'importance actuelle $d u$ pardon collectif

Le pardon qu'une personne accorde à une autre constitue une démarche difficile et complexe. Qu'en sera-t-il quand il s'agira d'accorder un pardon collectif, c'est-à-dire le pardon d'une collectivité à une autre collectivité? La difficulté prendra alors des proportions de plus en plus grandes à mesure surtout que les circonstances de l'événement déclencheur se seront perdues dans le temps de l'histoire, et que la mémoire collective sera devenue encline à les déformer à sa convenance parfois en insistant trop sur certains points, parfois en en occultant d'autres.

D'aucuns soutiennent que le pardon collectif est une entreprise impossible. Aussi préconisent-ils de chercher à oublier simplement le passé, de compter sur le temps pour que les vieilles rancunes s'évanouissent et pour que les mentalité changent avec la venue de nouvelles générations. Les tenants d'une telle position ne tiennent pas compte cependant du fait que les souvenirs douloureux, tout inconscients qu'ils puissent paraître, sont susceptibles, après des années d'un sommeil apparent, de ressurgir d'une manière violente. Pour s'en convaincre, l'on a qu'à songer aux conflits ethniques qui déchirent l'ancienne Yougouslavie et le Rwanda; aux haines tenaces qui opposent différentes nations entre elles: Arméniens contre Turques, Palestiniens contre Juifs, catholiques contre protestants en Irlande du Nord, etc. Que l'on pense aussi à la résurgence des souvenirs d'injustices qui semblaient oubliés, mais qui refont surface surtout sous l'influence des média, en particulier à l'occasion de films historiques ou d'émissions de la télévision: Schindler's list (Spielberg, 1994) qui raconte la vie du camp de concentration des Juifs à Varsovie; In the name of the father qui décrit les injustices subies par une famille irlandaise faussement accusée de terrorisme à Londres; la série de films sur les horreurs du Vietnam: Full Metal Jacket, Good Morning Vietnam et Platoon; les 
émissions de télévision sur les indiscrétions sexuelles: Les garçons de StVincent et $A u$ nom du Pere, etc.

En même temps que l'on constate une montée de réminiscences de malheurs communautaires, les demandes de pardon collectif tendent à se multiplier. Les évêques du Québec demandent pardon aux femmes pour avoir fait de l'obstruction au vote des femmes. Au cours de l'année 1992, on a vu le roi d'Espagne, Juan Carlos, demander pardon à la communauté juive pour le renvoi des Juifs d'Espagne à la demande de l'Inquisition cinq cents ans plus tôt. La même année, le maire américain de Salem organisa une cérémonie expiatoire pour condamner les accusations portées contre les "sorcières de Salem" ainsi que les tortures qu'elles ont subies. Au cours d'un pèlerinage au lac Saint-Anne dans l'ouest canadien, le 24 juillet 1991, les Pères Oblats ont demandé pardon aux autochtones pour avoir contribué à la destruction de leur culture à travers l'éducation donnée dans les écoles indiennes. Récemment, le premier ministre du Japon, Murayama s'excusait des horreurs commises par les soldats japonais sur le continent asiatique lors de la dernière guerre ${ }^{1}$. Des rumeurs circulent voulant que S.S. le Pape se prépare à faire l'an prochain une amende honorable aux nombreuses victimes de l'Inquisition.

Nos considérations présentent se répartiront en trois points: d'abord, nous établirons le fait de la mémoire collective et décrirons ses principales caractéristiques; puis nous explorerons les conditions essentielles d'un authentique pardon collectif; enfin, nous nous interrogerons à savoir qui est autorisé à accorder ou à demander un pardon collectif.

\section{Existence et transmission de la mémoire collective}

\subsection{La recherche}

Les milieux de la recherche sociale connaissent un intérêt renouvelé pour le phénomène de la mémoire collective comme phénomène social ${ }^{2}$;

1

Time 144/9 (August 29, 1994) p. 29.

2 En France, a paru l'ouvrage de G. NAMER, Mémoire et Société, Paris, Méridiens Klincksieck, 1987; l'auteur y présente l'œuvre de Halbwachs. La revue Esprit consacre également une partie du numéro de juillet 1993 au poids de la mémoire, à la question de la transmission du souvenir et du pardon dans l'histoire. Du côté anglophone, deux ouvrages collectifs démontrent un intérêt grandissant pour la mémoire collective: Collective Memory, édité par D. MIDDLETON et D. EDWARDS, Sage Publications, 1990 et Memory: History, Culture and the Mind, sous la direction de 
on prend conscience que la mémoire collective est une construction culturelle qui aide à comprendre les transformations d'ordre culturel. Ces recherches couvrent plusieurs thèmes tels que la transmission de la mémoire par les traditions orales; les contextes sociaux des souvenirs; les idéologies et les interprétations opposées du passé; les objets (musées, monuments,...) qui incarnent la mémoire collective; l'influence des médias; l'institution de la mémoire et de l'oubli, etc. Ces chercheurs effectuent leurs travaux dans la foulée de deux pionniers: le psychologue social anglais, F.C. Bartlett ${ }^{3}$ qui a étudié la mémoire comme processus social, et Maurice Halbwachs, sociologue français et disciple d'Emile Durkheim.

C'est en 1925 qu'a paru la première étude sur la mémoire comme phénomène social. L'ouvrage de Maurice Halbwachs Les cadres sociaux de la mémoire 4 marque un premier pas dans la reconnaissance des facteurs sociaux responsables des constructions opérées par la mémoire individuelle. Celle-ci a besoin de se référer à des représentations et des mémoires rassemblées par les groupes sociaux. Pour comprendre la mémorisation personnelle, il faut replacer l'individu à l'intérieur de ce carrefour des réseaux de solidarités multiples dans lesquels il est engagé. Dans La mémoire collective $(1950)^{5}$, Halbwachs fait une distinction importante entre "mémoire historique", qui est construction cristallisée et savante du passé, et "mémoire collective" qui est référence mouvante, parfois magique et mythique, souvent légendaire au passé. Il aura ouvert aussi des perspectives sur les représentations du temps variant selon les groupes sociaux. Pensons, par exemple, au temps religieux ou au temps profane; la "tradition" religieuse rythme autrement le changement que ne le suggère la succession rapide des événements profanes.

Au Québec, le sociologue Fernand Dumont, poursuivant sa recherche dans une perspective analogue, a abordé le thème de la mémoire historique comme une pratique sociale de l'interprétation de la société globale,

T. BUTLER, Londres, Basil Blackwell, 1989. Aussi la revue Communication 11, 1989, qui porte sur la mémoire sociale.

3 F.C. BARTLETT, Remembering : a Study in Experimental Social Psychology. Cambridge : University Press, 1932.

4 M. HALBWACHS, Les cadres sociaux de la mémoire. Paris, PUF, 1925.

5 M. HALBWACHS, La mémoire collective. Paris, PUF, 1950. 
à côté de l'idéologie et de la littérature ${ }^{6}$. "Les traditions historiographiques, écrit-il, permettent aux membres d'un groupement ou d'une société globale de disposer d'une définition existentielle de la communauté qu'ils forment leur offrant une image du destin qui les rassemble" 7 .

L'historiographie prend ainsi le relais des stratégies de groupes sociaux pour donner une interprétation de la société globale. Dumont a aussi montré, à propos du fait canadien-français, comment la mémoire historique a contribué à définir, par la survivance, une collectivité incapable d'harmoniser ses traditions nationales et son projet politique ${ }^{8}$.

La recherche récente explore divers thèmes: elle vise à déterminer les processus collectifs par lesquels les mémoires collectives des sociétés se construisent, se maintiennent et se transforment. De plus, elle tente de mieux discerner les sortes de mémoires sociales: il y a des mémoires publiques, des mémoires populaires, des mémoires familiales, des mémoires collectives... Enfin, la recherche essaie de comprendre comment la mémoire collective influe sur les pratiques politiques et culturelles d'une époque donnée.

Middleton et Edwards ${ }^{9}$ ont observé comment, dans les conversations courantes, sont transmises les représentations du passé et les constructions des événements passés. Prennent place alors les processus suivants de communication: la reconstitution d'un contexte à laquelle les participants au discours peuvent contribuer; le recours à la mémoire comme métaconnaissance dans les situations de doute ou de conflit d'interprétation. Ainsi, la reconstitution des événements passés de ces discours est soumise à une rhétorique sociale serrée. La version acceptée aura été mise à l'épreuve de cette rhétorique, sans quoi elle ne sera pas socialement acceptéc. Il en est de même pour l'expression émotionnelle par rapport aux événements. Elle est soumise à l'approbation du groupe. Des mécanismes de groupe comme l'accord, le désaccord, le sarcasme, l'embarras, conditionnent l'admissibilité des émotions de l'individu. Les auteurs ont aussi remarqué que le recours au passé fait partie d'une action sociale plus vaste; les

6 F. DUMONT, Chantiers. Essai sur la pratique des sciences de l'homme, Montréal, Hurtubise, HMH, 1973, et Genèse de la société québécoise, Montréal, Boréal, 1993.

7 F. DUMONT, Chantiers, p. 86.

8 F. DUMONT, Genèse de la société québécoise.

9 "Conversational remembering: a social psychological approach», dans Collective Memory, (D. MIDDLETON et D. EDWARDS, éd.), p. 23-45. 
versions collectives variées d'un événement sont fonction de leur utilité sociale ${ }^{10}$.

Peter Burke, de son côté, a étudié les modes de transmission de la mémoire sociale 11 . Il en a identifié cinq: les traditions orales; les mémoires et documents écrits dont les manuels d'histoire; les représentations visuelles et les monuments; les rituels de commémoration; les localisations historiques. Il note aussi que tous ces modes de transmission du passé peuvent être affectés par le phénomène de "légendarisation» ou de mythification de certains personnages ou événements du passé.

\subsection{Contexte social de la mémoire collective.}

Les sociétés traditionnelles ne se posaient pas le problème de la mémoire. Elles étaient conduites par des «traditions". Les manières de vivre, les croyances, la culture, reçues des ancêtres, avaient contribué à leur donner une vision stable du monde à laquelle on ne pouvait se soustraire sans mettre en cause toute la société. La consistance même de la vie collective reposait sur la transmission fidèle de cette vision du monde héritée des anciens. Ce qui ne veut pas dire que la mémoire était toute puissante. Même si pour bâtir l'avenir, il fallait toujours tenir compte des traditions garantes de la consistance de la vie collective, des conflits, des remises en question, des luttes pour le pouvoir et pour l'interprétation de la réalité par différents groupes étaient toujours possibles et donnaient lieu à des orientations divergentes pour l'avenir.

Les sociétés modernes au contraire reposent sur un tout autre postulat. Celui de la capacité de produire du nouveau. C'est la connaissance, la créativité, la technique, les communications qui façonnent la vie sociale. Il en résulte que la vie sociale est constamment en mouvance, en redéfinition d'elle-même; les groupes sociaux se font et se défont au gré des conjonctures. Les idéologies se succèdent plus rapidement que par le passé. Les événements se transforment sous la pression d'un monde en constante recréation de lui-même. Aussi faut-il en conclure que la Mémoire cède le

10 Nous retrouvons la même idée de l'influence du contexte social sur les souvenirs des individus dans A.J. MAYER, «Les pièges du souvenir », Esprit 193 (1994) 69.

11 P. BURKE, « History as social memory» dans Memory, (T. BUTLER, éd.), p. $97-113$. 
pas à la Politique qui a tendance à devenir l'instance déterminante de l'organisation sociale au lieu des traditions 12 .

Les traditions n'en continuent pas moins d'exister et de se transmettre, mais c'est comme si elles étaient discréditées, marginalisées. Elles sont reléguées au chapitre de la culture populaire ou du folklore. Pour cette raison, elles sont devenues étrangères à la rationalité qui régit des groupes désormais définis en univers sociaux plus consistants comme le monde du travail, de l'organisation, du savoir, de la politique, etc.

Dans ce nouveau contexte, la question de la mémoire collective et de son action se pose d'une autre façon. Les traditions qui sont discréditées, puis refoulées dans une sorte d'inconscient social ne sont-elles pas susceptibles de conférer un nouveau pouvoir à la mémoire collective? Ne seraitce pas là l'explication du réveil islamique de l'Iran qui, après s'être laissé tenter par le rêve américain sous le règne du Shah, est retourné avec force, voire violence, à ses racines coraniques? Pour s'en convaincre davantage, il suffira d'évoquer l'émergence de multiples conflits issus de souvenirs que l'on croyait effacés à tour jamais: le réveil des amérindiens qui se nourrit du souvenir de leurs vicissitudes, ce qui les justifie d'accuser les blancs d'intégration ethnique; la révolte des "enfants de Duplessis", dont la mémoire ne s'est pas laissée endormir par des mesures bureaucratiques; la résurgence inattendue du souvenir des abus sexuels.

\section{3 "Mouvement de la Mémoire»}

La mémoire collective, après avoir été discréditée au profit des idéologies du progrès, se relève de ses cendres. Des groupes sociaux y puisent de plus en plus leurs raisons de lutter pour leur libération. C'est pourquoi la transmission de la mémoire des blessures du passé est de plus en plus organisée et délibérée. D'où les revendications faites au nom du passé et les demandes de réparation.

12 Voir les réflexions de F. DUMONT, Le lieu de l'homme; la culture comme distance et mémoire, Montréal, HMH, 1968. «Si les traditions cèdent le pas à l'histoire, si les agglomérations synthétiques de la durée obligent à une lecture de plus en plus complexe de l'événement, le vide laissé par les modèles anciens doit être largement comblé par la décision politique. Par ailleurs, il se constitue des aires de plus en plus vastes de la vie où les significations prolifèrent dans toutes les directions, attendant en somme d'être caprées dans de nouvelles formes de la condition humaine et d'être canalisées vers des fins collectives qui restent encore à définir $»$ (p. 221). 


\section{À propos de la résurgence spontanée de la Mémoire, Arno Mayer écrit:}

La mémoire est sans nul doute à la mode en ce moment, tant à Caen qu'à Jérusalem, à Washington, à Moscou, à Varsovie, à Berlin, à Oradour-sur-Glane ou sur l'île de Gorée. Elle est devenue un produit de consommation qui rapporte et que l'on utilise à des fins politiques. C'est aussi la dernière mode chez les historiens occidentaux qui sont nombreux à étudier les commémorations, les mémoriaux et les musées. Nous sommes aujourd'hui moins hantés par le spectre d'un homme sans mémoire que par celui d'un homme sans oubli... 13

Pour reprendre l'expression de Thomas Butler, il existe dans le monde actuel un «Mouvement pour réhabiliter la Mémoire» 14 . Ce mouvement est illustré par la peur des Juifs d'oublier l'holocauste s'ils s'avisaient de pardonner; par le procès de Klaus Barbie en France; par la controverse autour du président Waldheim accusé de participation au judéocide; par le rappel des crimes de Staline contre les peuples de l'URSS et de la Pologne; par la reconnaissance récente par les États-Unis de traitements injustes commis contre les américains japonais au début de la seconde guerre mondiale; par la reconnaissance du gouvernement Australien de crimes perpétrés contre les aborigènes pendant le peuplement du pays. Il y a de plus en plus d'écrits sur le sujet. Ce mouvement, selon Butler, sourd de l'âme des peuples en vue de favoriser l'avènement d'une paix mondiale que la politique ne réussit pas à procurer. À son avis, le but de ce mouvement est d'inciter à se rappeler le passé en vue de le guérir et de le faire avec un esprit ouvert et généreux. Car pour que les anciennes blessures guérissent, il faut les ouvrir à nouveau.

\subsection{La mémoire collective ancienne refoulée par la création de la mémoire récente dans la société}

La question du pouvoir et de la place de la mémoire collective se pose d'une façon particulière dans les sociétés modernes. Celles-ci, nous l'avons signalé, sont axées sur l'avenir; les traditions ne garantissent plus l'immuabilité de leur monde; tous les jours, des événements se produisent qui se bousculent les uns les autres et qui sont rapportés par les médias. Des groupes sociaux sont touchés, voire formés par ces événements. 
Comment dès lors gérer la mémoire collective; par quels mécanismes le faire? L'assassinat de Marcellus François à Montréal donne lieu chaque année à un rituel commémoratif; des minorités ne veulent pas laisser se perdre le souvenir de cette tragédie et veulent le faire partager à l'ensemble de la société. Le massacre perpétré à la Polytechnique de Montréal est aussi commémoré chaque année; les femmes en profitent pour raviver la mémoire de la violence masculine à leur égard. Des groupes sociaux se forment en communautés interprétatives et assument eux-mêmes la responsabilité de maintenir le souvenir d'événements pénibles pour leur communauté. Il y a donc là une sorte de compétition, de jeu d'influences et de pouvoirs, pour que soit entretenue, dans la conscience collective, la mémoire de tel ou tel événement. Burke note aussi le phénomène des conflits de mémoire:

Étant données la multiplicité des identités sociales, et la coexistence de mémoires rivales et de mémoires possibles (mémoires familiales, locales, mémoires de classe, mémoires nationales, etc.), il devient de plus en plus important de se demander qui doit se souvenir de quoi et pourquoi ${ }^{15}$.

\subsection{Les stratégies contre la mémoire collective}

En plus des conflits opposant la mémoire récente et la mémoire collective, notons l'utilisation de deux stratégies contre la mémoire collective: la stratégie de l'oubli et la stratégie de la mémoire identitaire. Le but de la première est de créer l'amnésie sociale, celui de la seconde consiste à réduire la mémoire collective à la seule mémoire des oppressions de toutes sortes, et cela en vue d'une action sociale qui vise souvent à maintenir l'esprit de vengeance.

\section{La stratégie de l'oubli}

Les nombreux cas de destruction de documents prouvent l'existence d'une opposition systématique à la mémoire de certains événements. Cette tactique, on la retrouve surtout dans les pays totalitaires qui veulent faire oublier leurs méfaits. Prenons, par exemple, les répressions commises sur la place Tiananmen en Chine pour mâter le mouvement en faveur d'une république populaire. Les pouvoirs ont empêché la diffusion à la télévision d'images de la répression; ils sont allés jusqu'à nier officiellement l'existence même de la répression. Plus encore, ils ont déclaré que toute 
tentative de propager des informations sur l'événement serait considérée comme un crime. Qu'il soit question des camps de concentration nazis, ou des massacres de Staline en Ukraine, ou encore du génocide arménien, dans tous ces cas, c'est la politique de la «démémorisation" systématique des faits qui est pratiquée par les régimes totalitaires soit en détruisant les documents, soit en réduisant au silence les témoins. Toute opposition à l'effacement de la mémoire est assimilée par les régimes répressifs à une action contre-révolutionnaire. Quant aux victimes, conserver à leur place le souvenir de ce qui est arrivé devient leur unique moyen de continuer de vivre dans la mémoire des survivants.

Pour contrer les stratégies de l'oubli, on voit s'organiser des stratégies du souvenir. Les Russes connaissent présentement une période de réappropriation de leur histoire contre la censure systématique pratiquée sous le régime communiste. Pour eux, passer de l'amnésie à la remémoration est une condition essentielle à la redécouverte de leur identité. L'écrivain russe Alexandre Solzhenitsyn s'est donné comme mission de le rappeler. Ses ouvrages (Une journée dans la vie d'Ivan Denisovich; Le premier Cercle, Le pavillon des cancéreux, L'archipel du Goulag) veulent dire la mémoire collective de son peuple. Jusque dernièrement, les autorités soviétiques lui ont interdit de les publier en URSS. Mais aux yeux de Solzhenitsyn, écrire la vérité historique devenait une question de survie des victimes dans la mémoire du peuple russe.

\section{La stratégie de création de la mémoire identitaire}

La deuxième entrave à la mémoire collective se trouve dans la manipulation de la vérité que les versions officielles ont fabriquée aux dépens de la vérité historique. Elle est le fait de groupes qui, pour qu'on leur fasse justice ou pour avoir raison de se faire justice, s'arrangent pour qu'on ne voit en eux que des persécutés et des opprimés. Ils emploient cette tactique pour que la mémoire collective prenne une orientation sectaire et revancharde.

Aujourd'hui, tant les spécialistes de la mémoire que ceux de l'histoire affirment qu'il n'existe pas de mémoire collective pouvant opérer longtemps d'une manière spontanée sans orchestration 16 . Il faut donc des anniversaires, des commémorations, des pèlerinages, des mémoriaux, tels que ceux de Douaumont érigés en mémoire des soldats français tombés pendant la première guerre mondiale, et de ceux de Yad Vashem en sou- 
venir des Juifs assassinés pendant la seconde guerre mondiale. Pour les mêmes raisons, l'historiographie ne peut pas être considérée seulement comme une pure entreprise scientifique. En vérité, elle aussi sert à générer des légendes fondatrices, à promouvoir des identités nationales, à soutenir des systèmes de croyances, à justifier des abus de pouvoirs 17 .

Mayer dénonce ainsi les manipulations de la mémoire: "Les innombrables conflits d'ordre nationaliste, ethnique et culturel qui éclatent aujourd'hui, écrit-il, et dont beaucoup sont attisés par la ferveur religieuse, seraient impensables sans le moteur d'une mémoire sociale instrumentalisée $(\ldots)$. Nombre de nos voisins sur cette terre sont menacés par l'éruption d'une mémoire sociale hautement sélective, sectaire et sacralisée. Alors que dans un passé récent, hommes et femmes mouraient 'pour la patrie'..., en cette fin de siècle, ils meurent "pour la mémoire" "18.

Nous pourrions apporter ici de multiples exemples: pensons seulement au cas des Serbes ${ }^{19}$, des nationalistes catholiques de l'IRA qui déclarent ouvertement leur volonté de "ne pas oublier et de ne pas pardonner " 20 . Que dire maintenant du souvenir savamment entretenu de la Shoah sur lequel François Bédarida écrit: "C'est une injonction pour le

P. BURKE, « History as Social Memory " dans Memory, (T. BUTLER, éd.), ch. v.

A. J. MAYER, «Les pièges du souvenir », Esprit 193 (1993) 55.

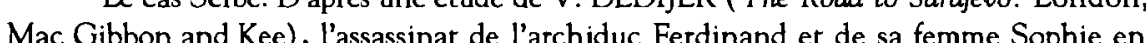
1914 serait motivé par la défaite des Serbes contre les Turcs à Kosovo en 1389. Cette défaite a valu des siècles de domination aux Serbes. Mais, dans leur mémoire, un événement a tenu lieu de vengeance: l'assassinat par le serbe Milos Obilic du Sultan Murad. Depuis, le régicide est devenu, pour les Serbes, une des plus haures valeurs ethniques. La mémoire de ces événements a été entretenue par les traditions orales, les poèmes, les chansons populaires. Quand l'Autriche a annexé la Bosnie en 1908, alors que les Serbes désiraient s'unir à la Bosnie, la réponse était inévitable. Quand l'archiduc d'Autriche s'est présenté à Sarajevo, le jour de l'anniversaire de la bataille de Kosovo, son assassinat par un Serbe a précipité le monde dans la première guerre mondiale. La mémoire d'un peuple entretenue par la poésie, les chants, les traditions orales, venait de renverser un empire. Certe mémoire des Serbes est encore à l'origine de leur agression actuelle contre les autres peuples de l'ancienne Yougoslavie; « les souffrances qu'ils infligent aujourd'hui ne seraient qu'une revanche pour celles qu'ils ont subies dans le passé, proche (la Seconde Guerre mondiale) ou lointain (les combats contre les Turcs musulmans) ». Citation tirée de T. TODOROV, «La mémoire et ses abus ", dans Esprit 193 (1993) 39.

20 Ibidem. 
peuple juif: «Zakhor!* Souviens-toi! On retrouve cette injonction 169 fois dans la Bible hébraïque. Une injonction que tout Juif entend tout au long de son existence " 21 .

La mémoire collective semble donc jouer un rôle primordial pour qu'une collectivité puisse se comprendre, se reconnaître et, pour certains, ne point perdre leur identité. Survient alors un grave danger quand une collectivité, au lieu de garder à vue toute son histoire, se fixe obstinément seulement sur les moments malheureux de son passé avec lesquelles elle tentera de s'identifier. C'est là que se durcissent les attitudes vis-à-vis du groupe offenseur et de ses descendants. En l'occurrence, le pardon s'avère impossible, car pardonner équivaudrait, dans cet esprit, à se nier soi-même en tant que collectivité et dès lors à disparâitte. Serait-il possible de penser à pardonner sans d'abord avoir corrigé une mémoire collective devenue en l'occurrence une "mémoire identitaire", par une mémoire historique véridique?

\subsection{Différences ethniques concernant la mémoire collective}

Peter Burke a remarqué que le degré d'attachement à la mémoire collective varie d'une culture à l'autre. Les Anglais ont une mémoire relativement courte des faits sociaux; ils préfèrent oublier le passé; ils souffrent facilement d' «amnésie sociale». Par contre, les Irlandais et les Polonais réagissent encore très fortement au rappel de faits qui remontent aux temps de Cromwell et de Napoléon. Les Indiens accordent peu d'importance au passé, alors que les Chinois y sont très attachés. On explique aussi que la fidélité de la mémoire au passé varie selon que l'on a été vainqueur ou vaincu. Les vaincus auraient la mémoire plus longue.

De même, la réanimation de la mémoire collective s'intensifie quand une culture est menacée; la réaction spontanée est de remonter à ses origines pour y retrouver une raison d'affermir et de réaffirmer son identité. Le passé remémoré devient alors partie de l'identité collective que l'on veut retrouver et maintenir.

\section{Les conditions essentielles pour réaliser un pardon collectif}

Trop souvent, on croit que le pardon individuel ou collectif résulte d'un simple élan de bonne volonté, presque magique, et n'exigeant aucune préparation. Nous voulons faire voir le contraire dans cette deuxième 
partie. La réalisation d'un pardon collectif se fait grâce à un long travail de transformation intérieure et de dialogue selon des étapes précises. Aussi, nous proposons de décrire ici une démarche de pardon qui favorise la guérison, le dialogue, la remise de la dette et éventuellement la réconciliation. Voici ces étapes et leur description.

\subsection{La reconnaissance officielle de la blessure et de la culpabilité}

\section{Reconnaissance de l'offense et de la blessure subies}

Les spécialistes du pardon se montrent unanimes sur le principe qu'il faut à la fois surmonter la tentation à l'oubli pour reconnaître officiellement l'offense et la blessure collectives avant de poser l'acte de pardonner. Luis Perez Aguirre définit comme suit le rôle de la mémoire collective dans une société traumatisée par des injustices: «Ici apparaît le rôle de la mémoire collective. Il ne s'agit pas de juger pour cela, il y a les juges et l'ordre constitutionnel, mais de protéger le présent qui ne peut se concevoir sans l'histoire immédiate». Il ajoute: "Il est indispensable de recourir à la mémoire pour nous reconnaître, reconnaître ce qui est nôtre, le découvrir dans cet amalgame de douleurs et d'espérance " 22 . Cette nécessité du souvenir de l'offense pour guérir, Miguel Angel Estrella, ce pianiste argentin torturé durant le régime militaire de son pays, la confirme en ces termes: "Il faut une mémoire des sociétés. Cela fait partie des racines d'une communauté humaine, et les racines, plus elles sont profondes et authentiques, plus l'être humain peut voler haut ${ }^{23}$.

\section{Le danger de l'oubli}

Le danger qui guette ceux qui oublient leur passé malheureux, c'est de rester contaminés, à leur insu, par les effets mauvais de l'offense, et de reproduire chez les autres le mal dont ils ont été eux-mêmes victimes. Olivier Abel fait appel à la mémoire pour conjurer ce danger: «Tant que l'on n'a pas rompu avec l'oubli, avec la loi du silence, du refoulement des plaintes et des colères, le passé oublié est toujours présent, il se répète, il se reproduira encore. L'horreur n'est pas finie parce qu'elle est «oubliée», elle se poursuivra indéfiniment tant qu'une parole n'aura pas rompu avec l'oubli et accepté de faire mémoire. Car c'est en reconnaissant ce qui s'est

22 L. P. AGUIRRE, «Mémoire, justice et pardon», Spiritus, la réconciliation, conflits, justice et pardom. 135, tome xxxv, (1994) 151.

23 M. A. ESTRELLA, * Tu es des milliers», Esprit 193 (1993) 176. 
passé que l'on rompt avec la continuation du passé dans le présent» 24 . Il n'existe donc pas réellement une chose telle que l' "oubli " définitif d'un traumatisme, car la transmission inconsciente de celle-ci demeurera toujours active influençant d'une manière sournoise ses façons de voir le monde et les autres.

\section{La transmission de la blessure}

Pour illustrer la transmission inconsciente d'une blessure, nous étudierons le phénomène de la mémoire transgénérationnelle d'une famille. Nous pensons qu'il est légitime d'extrapoler les résultats de cette recherche à la mémoire d'une communauté.

Comment expliquer l'existence dans une famille de comportements déviants tels que revendications exagérées, attitudes malsaines (méfiance, gêne, sentiment d'infériorité et besoin exagéré de domination, etc.), tendances suicidaires, traditions d'inceste et autres? Le créateur de la therapie contextuelle, le psychiatre Ivan Bozsorminyi-Nagy, attribue l'origine de ces comportements destructeurs et autodestructeurs à des traumatismes encourus par suite d'événements mal vécus par les ancêtres d'une famille. Ces blessures familiales seraient transmises d'une manière imperceptible par des secrets de famille d'une génération à l'autre. Refoulés dans l'inconscient familial, les souvenirs de ces événements pénibles qui n'ont pas pu être liquidés par la parole et le deuil, continuent de hanter les descendants.

Ce thérapeute soutient que les systèmes familiaux sont commandés par des "loyautés invisibles", à savoir un sens aigu non exprimé de la justice qui se traduit par le souci inconscient de tenir une rigoureuse comptabilité des dettes et des mérites qu'une famille a accumulés. Ce besoin incompressible de justice serait transmis d'une génération à l'autre, et deviendrait l'élément déterminant des systèmes familiaux. Ces «loyautés invisibles " transmises d'une génération à l'autre et se reflétant dans des comportements bizarres, continueraient, d'une façon, à se faire justice et à trouver compensation pour les injustices subies dans le passé 25 . C'est comme si on se passait d'une génération à l'autre, un "grand livre de compte " dans lequel auraient été enregistrées les actions justes ou injustes commises par les membres d'une famille de générations en générations. in intergenerational family therapy. New-York, Harper and Row, 1973. 
Quand les «loyautés invisibles» ne sont pas respectées, à savoir que les actes justes ne sont pas récompensés et les actes injustes punis, il en résulte pour les générations subséquentes des comportements pathologiques: rigidité, revendications irrationnelles, actions autodestructrices, etc...

Si une blessure non guérie peut se transmettre ainsi d'une génération à l'autre d'une manière non consciente, n'est-il pas légitime de présumer que le même phénomène peut fort bien se produire dans la vie d'un groupe ou d'une collectivité? Il s'ensuit que les membres de cette collectivité seront susceptibles de souffrir de symptômes pathologique sans même se douter qu'ils en ont hérités. D'où la difficulté toute spéciale que présente cette situation: comment guérir d'une blessure dont on ignore l'existence et pardonner une offense dont on ignore l'origine ? ${ }^{26}$

\section{Reconnaissance de la culpabilité de l'offense}

Pour la guérison des victimes, il est important que l'offenseur reconnaisse ouvertement sa responsabilité et sa culpabilité vis-à-vis l'offense. Les victimes se voient confirmées dans leur conviction intime qu'une injustice a été commise à leur égard, et se sentent justifiées d'exprimer leurs émotions de colère et de tristesse.

Certains prétendront que c'est utopique d'attendre des bourreaux l'aveu de leur culpabilité. Pourtant il y a des faits qui prouvent le contraire. Pensons aux chefs de communauté et même d'État qui récemment ont demandé publiquement un pardon (voir les exemples cités dans l'introduction de l'article). Et il semble bien que ce mouvement mondial actuel de faire amende honorable pour les fautes passées d'une collectivité ira en s'accentuant.

Par contre, il arrive trop souvent qu'on essaie de gommer la culpabilité d'un groupe donné sous prétexte que les deux parties seraient coupables dans l'affaire, que les coupables ne faisaient qu'obéir à des ordres, ou encore qu'il est inutile de forcer des aveux de l'un ou de l'autre partie sans s'embourber dans une série de justifications. Certes, l'idéal serait que les bourreaux reconnaissent leurs fautes et en demandent pardon. Mais à défaut de le faire, rien n'empêche les victimes de poursuivre leur travail de guérison afin de se disposer à pardonner.

26 Pour poursuivre la recherche concernant la transmission transgénérationnelle d'une blessure, il est intéressant de lire le travail de A. ANCELIN SCHÜTZENBERGER, Aie, mes aïeux! Paris, La Méridienne, 1993. 


\section{2 «Se désidentifier» de la mémoire identitaire}

La sociologie parle de plus en plus de "mémoire identitaire». Celleci serait la "remémoration" obstinée, par une groupe, d'une offense subie qui l'autoriserait à se poser en perpétuelle victime. C'est une façon pour lui d'affirmer une identité. Aussi Olivier Abel remarque que le souvenir d'un malheur commun est plus susceptible de pouvoir définir la personnalité d'un groupe que la mémoire d'un succès ou d'un bonheur: "la mémoire obsédante d'une faute commune ou d'une souffrance commune structure mieux une identité communautaire que la mémoire d'un bien ou d'un bonheur commun; malheureusement. Mais cette identité refusera toute rétribution, tout pardon, tout effacement qui pourrait la modifier: ancrée dans une dette irréversible, elle ne voudra que l'entretenir; elle fera tout pour la reproduire, la continuer 27 . L'attachement obstiné à la mémoire identitaire entraîne souvent un étrange revirement: les victimes se font les bourreaux à leur tour: «...les peuples qui ont le plus souffert deviennent souvent les plus impitoyables» 28 .

Todorov plaint le groupe qui, sous l'influence de démagogues, ravive sans cesse les souvenirs douloureux s'empêchant d'effectuer, par le moyen du deuil, une catharsis bénéfique:

Le groupe qui ne parvient pas à s'arracher à la commémoration lancinante du passé, d'autant plus difficile à oublier qu'il est douloureux, ou ceux qui, au sein de ce groupe, l'incitent à vivre ainsi, méritent moins la sympathie: cette fois-ci, le passé sert à refouler le présent, et ce refoulement-là n'est pas moins dangereux que l'autre. Tous ont le droit de recouvrer leur passé, mais il n'y a pas lieu d'ériger un culte à la mémoire pour la mémoire; une fois le passé rétabli, on doit interroger: à quel usage servira-t-il ? ${ }^{29}$.

Ceux qui aimeraient se renseigner davantage sur les difficultés résultant d'une mémoire identitaire, nous les renvoyons à l'excellent article déjà cité d'Arnou Mayer "Les pièges du souvenir». Pour l'illustration des cas actuels de mémoire identitaire, nous référons à Howard Stein qui a analysé la mémoire du judéocide ${ }^{30}$, ainsi qu'au cas de la mémoire du

O. ABEL, « Ce que le pardon vient faire dans l'histoire », Esprit 193 (1993) 70. Ibid., p. 67.

29 Ibidem.

30 H.F. STEIN (éd.), "The holocaust, the uncanny, and the Jewish sense of history », Political Psychology 5/1 (1984). *Depuis l'alliance (*akedah ») abrahamique avec Dieu, conduisant au sacrifice d'Isaac, jusqu'à l'alliance mosä̈que dans laquelle 
génocide arménien laquelle est exploitée surtout aux fins de regrouper les Arméniens de la diaspora ${ }^{31}$.

Le travail du deuil et l'élaboration d'un pardon deviennent impossibles tant et aussi longtemps qu'une collectivité ne se détache pas de sa mémoire identitaire et de son désir de vengeance. Pour une description plus complète des méfaits du ressentiment et de la vengeance, on voudra bien se référer à notre ouvrage sur le pardon 32 .

L'acte d'abandonner la mémoire malheureuse constitue un point tournant dans le processus de deuil. Il représente un défi énorme de détachement de soi. Il s'agit pour un groupe qui s'est identifié à son destin douloureux de savoir comment accepter de renoncer à s'identifier à la mémoire malheureuse sans la nier? Une collectivité qui prendra conscience de tous les dommages psychologiques, sociaux et spirituels qu'elle s'est infligée en s'identifiant à la mémoire identitaire, trouvera l'énergie de prendre ses distances par rapport à celle-ci. Elle sera alors à même de vivre son présent, d'entretenir des projets d'avenir faits de tolérance, voire de convivialité avec ses «vieux ennemis».

Israël devient (avec hésitation, révolte et assiduité) le Serviteur de Dieu et jusqu'à présent, les Juifs ont accepté l'attitude passive, féminine envers Dieu. Par extension, les Juifs ont accepté l'attitude féminine, passive envers “l'histoire», c'est-à-dire, qu'ils continuent d'expérimenter l'histoire comme un continuum de souffrances qui leur arrivent «à eux», qu'ils doivent porter. Ainsi, au lieu de présenter tout doucement l'autre joue aux persécuteurs, les Juifs projettent la cause de leur agression contre euxmêmes (le super ego sadique) sur les gentils, qui correspondent en agissant comme des délégués du super ego sadique et persécutent l'ego masochiste " (Ibidem, p. 24). L'auteur émet l'hypothèse que les Juifs mettent l'accent sur leur vocation sacrificielle pour ne pas regarder (par projection et intellectualisation) le processus de victimisation des victimes elles-mêmes; l'idéologie sacrificielle prédispose les Juifs à jouer un rôle historique de partenaires victimisés dans un système social sadomasochiste gouverné par des identifications projectives réciproques.

31 Olivier Abel, au cours d'un entretien avec un Turc et un Arménien, résume ainsi l'impasse où se trouvent Arméniens et Turcs : "Ce qui me frappe dans ce que vous avez dit l'un et l'autre, c'est le sentiment que l'identité arménienne est organisée autour d'une mémoire du génocide (mémoire identitaire et non mémoire historique), et le sentiment que l'identité turque est plutôt fondée sur une amnésie. »O. ABEL (éd.), "Différend, entretien avec Haldun Bayri et Anabide Ter Minassian ", dans Le pardon, briser la dette et l'oubli, (Séries morales, 4), Paris, Autrement, 1991, p. 163.

32 J. MONBOURQUETTE, Comment pardonner? Ottawa, Novalis, 1992, p. $88-90$. 
Mais que dire maintenant des injustices subies? Devrait-on toujours exiger que la dette soit reconnue et réparée avant de pouvoir poursuivre sa démarche de pardon?

\subsection{Le partage des responsabilités et les exigences de la justice}

Pour entrer véritablement dans la dynamique d'un pardon collectif, il faudrait commencer d'abord par satisfaire aux exigences de la justice. Car le pardon, loin de dispenser du devoir de rendre justice, exige au contraire que justice soit faite. Il entre en jeu au moment seulement où, sur le plan de la justice, il ne reste plus rien à faire.

D'entrée de jeu, le pardon est un acte de courage, car il exige du pardonneur qu'il voit à faire cesser les gestes offensants et injustes à son égard. Avant même de penser à pardonner, un groupe doit d'abord commencer par faire respecter ses droits. Nous pensons ici, par exemple, au devoir que les catholiques de l'Irlande du Nord ont de réclamer d'une manière non violente un traitement équitable sur le plan social, politique et économique. Nous insistons sur l'importante nécessité d'adopter une attitude de non-violence dans la revendication de ses droits, sinon on dévira dès le départ de la trajectoire du pardon. Toute tentative de corriger les injustices d'une manière violente ne fera qu'aggraver le conflit et éloigner toute possibilité de pardon et, par la suite, de réconciliation.

Dans le même ordre d'idées, nous croyons qu'avant même de mettre toutes ses énergies à vouloir pardonner, il faut s'appliquer à corriger les situations injustes que les évêques d'Amérique latine à Médelin et à Puebla ont qualifiées de "réalités mauvaises" ou de "structures de péché». Décrivant la situation dans ces pays, Jon Sobrino reprend des déclarations des évêques: "Bien qu'elles soient connues, il faut répéter leurs affirmations fondamentales. La réalité latino-américaine est dans "une situation de péché" (Medellin, 'Paix', 1), exprime "une situation de péché social" "(Puebla, 28) ${ }^{33}$. Vouloir pardonner dans une société où l'injustice s'est établie dans ses structures mêmes sans constater une réelle volonté de changement équivaudrait à une démission. Le pardon ne s'impose qu'après avoir pris tous les moyens pour que justice soit faite.

Par contre, est-il possible, dans chaque cas de conflits historiques entre collectivités, de départager les responsabilités et d'inviter chacune des parties à reconnaître les siennes et à se disposer à accomplir une juste 
réparation? Malheureusement de telles prises de conscience sont rares; ce qui ne veut pas dire qu'on doive renoncer d'emblée à cet idéal.

Plusieurs auteurs soutiennent que, dans la plupart des différends historiques, il est impossible d'en arriver à répartir les responsabilités et, par voie de conséquence, à demander de faire réparation. Abel montre bien cette impossibilité de faire la lumière sur les tenants et aboutissants d'un conflit historique. C'est ce qu'il appelle "le tragique de conflit» et "le tragique de l'irréversible». Pour lui, certains différends sont insolubles parce qu'en l'occurrence le dialogue s'avère impossible, et cela à cause des "dénis et des visées incompatibles", de la mémoire identitaire, "des justifications antagonistes", des langages divergents appartenant à des mondes différents, enfin en raison de visions de l'histoire qui ne peuvent pas se rejoindre ${ }^{34}$.

À cause de ce qu'il nomme "l'étroitesse d'engagement des protagonistes, c'est-à-dire leur incapacité de changer de point de vue " 35 , Abel suggère de renoncer à chercher à répartir les responsabilités dans le cas d'un conflit historique et à exiger des réparations. Au lieu de faire le procès d'une époque révolue et de vouloir distribuer les culpabilités, il conseille plutôt aux antagonistes de se mettre d'accord sur l'existence entre eux d'un désaccord presque total et insurmontable. Ainsi, ils pourront dialoguer en faisant abstraction de leur incapacité de faire la lumière sur le passé. Devant une situation aussi impossible, il propose pour le contourner de recourir à une «sagesse pratique» que nous décrirons plus loin.

Même si la situation est telle qu'il s'avère impossible de satisfaire à toutes les exigences de la justice et de la vérité, il sera toujours possible d'envisager la possibilité de s'entendre et de se réconcilier. N'est-ce pas ce genre d'attitude qui a permis récemment de conclure des accords entre Palestiniens du FLP d'Arafat et l'État d'Israël du président Rabin, ou encore d'effectuer des changements radicaux de la politique en Afrique du Sud grâce aux interventions de l'ex-président de Clerk et de Nelson Mandela? 


\subsection{Possibilité de reviure la blessure occasionnée par la perte et capacité de faire son deuil}

Plus d'un auteur affirment la nécessité de «faire son deuil» pour en arriver à pardonner. Tzvetan Todorov décrit la situation malheureuse de ceux qui ne peuvent "lâcher prise sur le passé" :

L'individu qui ne parvient pas à accomplir ce qu'on appelle le travail de deuil, qui ne réussit pas à admettre la réalité de sa perte, à s'arracher au choc douloureux qu'il a subi, qui continue de vivre son passé au lieu de l'intégrer dans le présent, qui est dominé par le souvenir sans pouvoir le domestiquer (et, c'est, à des degrés divers, le cas de tous ceux qui ont vécu dans les camps de la mort), cet individu est évidemment à plaindre et à secourir: il se condamne involontairement lui-même à la détresse sans issue, sinon à la folie ${ }^{36}$.

Par ailleurs, la plupart des auteurs qui affirment la nécessité de faire son deuil du passé ne semblent pas bien comprendre tout ce que le deuil exige de victoire sur le déni et de catharsis émotionnelle. Même certains, comme Olivier Abel, confondent indûment deuil et pardon: «En désignant l'irréparable, l'intraitable, ce qu'on ne peut raconter entièrement, le pardon (conformément à son étymologie probable) accepte qu'il y de la perte, des dettes qui ne sont plus des dettes, des possibles qui ne sont plus des possibles. Il fait ce travail de deuil sans lequel il n'y a pas de travail d'enfantement ou de résurrection possible d'un autre présent 37 . Or faut-il le répéter, le deuil tout en étant une étape nécessaire au pardon, se distingue nettement de celui-ci.

À première vue, il paraît plus facile d'amener un individu qu'un groupe à faire son deuil. Par contre, un rituel de deuil fait en groupe et bien mené possède un puissant effet d'entraînement; il permet à une foule de se donner des modes acceptables d'expression de sa tristesse, de sa culpabilité et même de sa colère. Des journées nationales de deuil bien orchestrées peuvent produire des effets de purgation émotionnelle collective. Pensons, par exemple, au rituel de deuil national qui fut organisé lors de la mort du président de la Corée du Nord, Kim Il Sung, rituel au cours duquel on a pu voir des milliers de ses sujets le pleurer

T. TODOROV, «La mémoire et ses abus », Esprit 193 (1993) 41.

O. ABEL, « Ce que le pardon vient faire dans l'histoire », Esprit 193 (1993) 70. 


\subsection{Trouver un sens à son drame historique}

Faire son deuil avant de pardonner est donc un passage obligé. Mais la catharsis de groupe suffit-elle elle-même à rendre un deuil à terme? Afin que le deuil ne laisse pas dans l'âme des relents d'humiliation et des sentiments d'amoindrissement de son être, un individu ou un groupe a besoin de trouver ou de donner un sens à la souffrance infligée et à l'injustice subie.

Au lieu de rester prisonnier d'une "mémoire identitaire" qui conditionne son être et son agir futur, le groupe qui réagit à son malheur et qui parvient à donner un sens à sa souffrance, récupère le meilleur de luimême et redécouvre sa mission dans la vie. Pour y parvenir, il aurait avantage à s'inspirer de la logothérapie créée par le psychiatre Viktor Frankl. Cette méthode thérapeutique offre justement aux victimes le moyen de donner un sens positif à leur drame et du même coup, à dépasser leur état de victime ${ }^{38}$.

Ainsi, pour trouver ou donner une signification positive à son malheur, il est recommandé de se poser certaines questions. En voici quelques unes en guise d'exemples: Quel sens dans l'ensemble de votre vie donnez-vous à votre malheur passé? Qu'avez-vous appris de lui sur vous-mêmes? De quelles ressources insoupçonnées avez-vous pris conscience à la suite de votre drame? Ces questions, un individu peut y répondre lui-même, mais lorsqu'une collectivité se trouve concernée, il appartient à ses sages et à ses poètes de le faire en son nom.

Examinons maintenant quelques réflexions de spécialistes sur la manière de donner un sens à un malheur collectif. Parlant du judéocide, Mayer propose de donner à la Shoah une portée universelle: "Transmettre le souvenir de la blessure oui, écrit-il, mais l'ouvrir sur l'universel, sur les blessures des autres; (...) ce n'est pas trahir les victimes du judéocide que de "désectariser», ou si l'on veut de "réuniversaliser" leur mémoire en l'éclairant par des aperçus historiques d'ordre homologique et contextuel» 39 .

38 V. FRANKL, Découvrir un sens à sa vie. Montréal, Les éditions de l'homme, 1988. Gordon Allport dans la préface décrit ainsi la logothérapie : « L'objet et le défi de la logothérapie, qui est une version du docteur Frankl de l'analyse existentielle moderne, est d'entrelacer les fils ténus d'une vie brisée afin d'en faire un modèle de sens et de responsabilité » (p. 7).

39 A. J. MAYER, «Les pièges du souvenir », Esprit 193 (1993) 59. 
Todorov, pour sa part, propose une démarche analogue pour réaliser un sens à un malheur collectif, après avoir distingué entre «mémoire littérale" et "mémoire exemplaire". La première n'est que le simple rappel de l'événement passé et de la blessure qui en résulta. La mémoire exemplaire retourne à l'événement passé pour y voir une illustration d'injustice et d'oppression. L'événement ainsi interprété fournit l'occasion et le courage de dénoncer d'autres situations d'oppression actuelles et similaires. Dès lors, la mémoire littérale qui est refermée sur elle-même par nature se transforme en une mémoire au service de la justice; elle devient du même mouvement une "mémoire exemplaire" tout en conservant fidèlement la version réelle des faits historiques. Par exemple, c'est ce genre de travail de transformation qui a amené un David Rousset, ancien déporté des camps nazis, à mener une offensive contre les camps soviétiques 40 .

Le même auteur résume comme suit sa pensée sur la transformation de la mémoire littérale en une mémoire exemplaire: «Ceux qui, à un titre ou à un autre, connaissent l'horreur du passé ont le devoir d'élever leur voix contre une horreur autre, mais bien présente, se déroulant à quelques centaines de kilomètres de chez eux. Loin de rester prisonniers du passé, nous l'aurons mis au service du présent, comme la mémoire - et l'oubli - doivent se mettre au service de la justice " 41 . Voila comment, grâce au recadrage d'une situation d'injustice, d'humiliation et d'oppression, les victimes du passé accomplissent une mission de salut en faveur des victimes actuelles.

\subsection{De quel pardon collectif s'agit-il?}

Lors d'une entrevue avec un Turc et un Arménien, Olivier Abel leur proposa de songer à la pratique du pardon chrétien en vue de solutionner le différend qui les séparait. La réaction de son interlocuteur arménien Adabide Ter Minassian ne se fit pas attendre. Il rappela que le pardon n'a sa place qu'à l'intérieur d'une éthique chrétienne, et que d'ailleurs il est presqu'impossible de l'exercer dans toutes les situations de conflits 42 . En réponse, Abel s'avisa de changer de langage, il proposa un pardon décrit en termes d'uacte d'autonomie éthique" ce qui équivalait

\footnotetext{
40 T. TODOROV, «La mémoire er ses abus », Esprit 193 (1993) $42-43$.

41 Ibidem, p. 44.

42 Ibidem, p. 163
} 
à faire dire au pardonneur éventuel son intention: «Moi, je brise le cercle de la vengeance; je ne me vengerai pas ".

Le pardon que décrit Abel s'éloigne de celui de la pensée chrétienne. On sait que dans une perspective chrétienne, le pardon est l'expression de l'amour parfait, si parfait qu'il mène jusqu'à l'amour des ennemis. Et la raison fondamentale d'un tel amour tire son origine de la croyance de la foi chrétienne que tous les hommes sont frères les uns des autres parce qu'ils ont tous Dieu comme père. En dehors d'un tel pardon chrétien, il ne reste plus comme possible que la formule d'un pardon sécularisé.

Comment peut-on définir le pardon sécularisé? C'est une notion de pardon qui, après s'être détachée de la tradition chrétienne, est devenue multiforme. À l'opposé de la définition positive du pardon chrétien, le pardon sécularisé se définit sous des formes négatives telles que "ne pas se venger», "ne pas faire payer la dette", «enlever le ressentiment en soi ", "oublier ", etc.

Ainsi donc, le pardon "sécularisé " ne se fonde plus sur l'amour de Dieu et du prochain, mais sur la peur: peur de tomber dans une spirale de violence, d'obéir à "la fatalité du sang", peur de la répétition insensée de la destruction mutuelle, peur de stagner dans un passé malheureux qui dévore le présent et bloque tout projet d'avenir.

Pour contourner toutes ces peurs, Olivier Abel propose d'exercer un pardon qui ressemble à un pardon sécularisé tout en conservant des réminiscences religieuses. Ce pardon s'articule sur deux charnières: «briser l'oubli * et « briser la dette.

C'est cette double rupture, avec l'oubli et avec la dette, qui fait du pardon quelque chose de plus difficile qu'un simple oubli ou qu'un ressentiment déguisé. Il faut le répéter: entre les deux écueils, la voie du pardon est étroite et pour dire impossible: quelle pourrait être cette parole assez extraordinaire pour discerner l'oubli vital de l'amnésie facile, pour transformer cette amnésie douloureuse en mémoire vivante, et pour effacer la mémoire malade et obsédée du ressentiment? ${ }^{43}$.

Mais essayons de voir plus dans la nature d'un tel pardon. Nous en reproduirons ici les traits majeurs que l'auteur en donne. 
Ce pardon est le fruit de cette «sagesse pratique» que décrit Paul Ricœur dans son ouvrage Soi-même comme un autre, (Paris, Seuil, 1990). Cette sagesse invite à construire le pardon sur un compromis, à savoir que les adversaires acceptent d'être d'accord sur le fait qu'ils ne seront jamais d'accord sur la répartition des responsabilités et des culpabilités dans le conflit, ni sur le partage des dettes encourues.

Ce pardon se fonde donc sur la décision d'abandonner de part et d'autre la mémoire identitaire: "C'est-à-dire qu'il repose sur un désistement réciproque, sur l'acceptation par le protagoniste de sa disparition en tant qu'identique à lui-même, sur le consentement du pardonnant comme du pardonné à devenir autre que lui-même " 44.

Ce pardon va au-delà de toutes les exigences de l'éthique, car celle-ci n'est pas habiletée à prescrire le pardon. Il faut alors recourir à une justice "supérieure" qu'Abel préfère désigner du nom de «justesse", une qualité morale qui rend sensible au besoin de pardonner. Cette qualité de "justesse" serait un écho lointain de propos bibliques où il est question de rendre quelqu'un "juste" au-delà de toute justice.

Ce pardon résulte donc de cette sagesse pratique que met en activité l'imagination afin qu'elle invite les antagonistes à se placer au-dessus des torts et des conflits (le tragique de conflit) et au-delà des revendications de réparations, impossibles d'ailleurs à faire (tragique de l'irréversibilité). Abel décrit ce travail de l'imagination en ces termes: "La sagesse du pardon consiste à accepter qu'il n'y ait pas de dernier mot, pas de langage complètement commun, et à tisser entre les deux histoires ou deux mémoires incompatibles, une sorte d'intrigue qui est celle de leur compromis, de leur cohabitation" 45 . L'effort d'imagination demandé aux antagonistes consiste donc à chercher à se mettre à la place de son vis-à-vis pour voir les choses de son point de vue et les vivre comme il les vit luimême. En «se mettre dans la peau de son adversaire», on se dispose soimême à lui reconnaître les mêmes droits que les siens.

Abel, qui préconise cette sorte de pardon n'est pas sans en pressentir toute la fragilité: «... les paroles qui énoncent le pardon, tout en refusant la clarté d'une situation où l'un serait le pardonnant et l'autre le pardonné, sont des paroles fragiles. Ce sont des paroles qui conjuguent des discours hétérogènes" 46 . Â la fin de cette étude sur le pardon, fruit de 
"la sagesse" pratique, nous ne sommes pas sans nous demander si ce genre de pardon n'est pas commandé en fin de compte par la peur des deux parties de se détruire mutuellement.

\section{Qui peut accorder le pardon collectif ou le demander?}

\subsection{Nul ne peut pardonner au nom des victimes à moins d'avoir reçu d'eux le mandat}

Les penseurs qui écrivent sur le pardon individuel et le pardon collectif s'entendent sur le principe que nul ne peut pardonner au nom des victimes. Aguirre va jusqu'à affirmer: "On ne peut pas pardonner au nom d'un autre, d'un tiers, sinon le pardon offert au bourreau devient une cruauté envers la victime " ${ }^{47}$. Qu'en est-il maintenant s'il ne s'agit non pas de pardonner, mais de demander pardon au nom d'autres? Encore ici, nul ne doit s'autoriser à se faire le porte-parole d'une communauté pour demander pardon s'il n'a pas consulté chacun des membres, et s'il n'a pas reçu d'eux le mandat explicite de le faire. D'ailleurs, demander pardon à la place d'un autre sans son autorisation équivaut à reconnaître et déclarer indûment la culpabilité de ce dernier.

Le 24 juillet 1991, les Supérieurs provinciaux Oblats du Canada s'autorisaient de faire amende honorable auprès des autochtones au nom des douze cents Oblats canadiens. Le méfait qu'ils confessaient, était que les Oblats auraient contribué à la destruction de la culture des autochtones à travers l'éducation donnée dans les écoles résidentielles indiennes.

Bien que plusieurs Oblats reconnaissaient l'opportunité de ce geste, beaucoup protestèrent pour deux raisons: tout d'abord parce qu'on ne les avait pas consultés pour faire en leur nom cette demande de pardon, et ensuite parce qu'en vérité ils ne se reconnaissaient pas coupables des crimes qu'on leur imputait dans la demande de pardon. D'autres protestations sont venues d'indiens eux-mêmes qui, loin de se considérer comme des victimes du système d'éducation des écoles résidentielles, en reconnaissaient la raison d'être et la légitimité pour l'époque.

Une première conclusion s'impose: ne jamais pardonner ou accorder un pardon ou demander pardon au nom d'une personne ou d'une collectivité sans leur approbation. Autrement, on risque d'engendrer plus de 
discordes que d'en résoudre 48 . La deuxième conclusion est claire: préparer avec beaucoup de discernement le motif de la demande de pardon afin de ne pas incriminer injustement des personnes concernées.

Ces conditions remplies, le pardon collectif bien conçu et bien préparé est appelé à produire des effets bénéfiques. Entres autres, il met les communautés en conflit sur la voie de la réconciliation. Nous pensons ici à la demande de pardon que l'évêque d'Ontario Peter Mason, titulaire de la cathédrale anglicane St-George de Kingston, a faite à sa communauté. L'organiste de la cathédrale avait été condamné pour attentat à la pudeur contre des enfants de la chorale, pendant plus de vingt ans, sans que les autorités de la cathédrale n'interviennent malgré les nombreuses dénonciations. Lors d'une cérémonie, des paroissiens avaient organisé une démonstration devant la cathédrale pour dénoncer le silence du clergé. Le nouvel évêque se faisant solidaire de ses prédécesseurs, assuma la responsabilité de la négligence de ne pas avoir corrigé plus tôt les abus. C'est à titre de représentant symbolique de toute l'institution de la cathédrale qu'il demanda pardon aux fidèles. De cette façon, il se rallia une grande partie des protestataires qui entrèrent dans la cathédrale pour assister à la cérémonie 49 .

\subsection{Les descendants de victimes peuvent-ils pardonner aux offenseurs de leurs parents ou ancêtres?}

Comme nous l'avons déjà affirmé, nul ne peut pardonner à la place d'une victime. Par ailleurs, les fils et les filles dont les parents ou arrière-parents ont été victimes, ne sont-ils pas eux-mêmes devenus victimes par suite des effets malheureux qui s'ensuivirent pour eux: pertes de biens matériels, pertes de chances d'éducation, pertes psychologiques telles que sentiment d'insécurité et de manque confiance, pertes spirituelles, etc. À notre avis, les individus qui appartiennent à une collectivité autrefois victime d'humiliation et d'oppression, ont le droit et le devoir de chercher à se guérir des atavismes et des traumatismes dont ils ont hérité. Certes, les descendants d'une collectivité qui fut blessée, ne peuvent pas pardonner au nom de leurs ancêtres, mais ils le peuvent à titre de victimes éloignées qui subissent encore le destin douloureux de leur communauté.

48 Ceux qui seraient intéressés à approfondir l'événement peuvent avoir accès à un dossier aux Archives Deschatelers, 175, rue Main, Ottawa, K1S 1C3.

49 On peut retrouver le texte de la demande de pardon dans le quotidien WingStandard, de Kingston, Monday, March 1, 1993. 


\section{Conclusion}

Avec l'avènement "du mouvement de la mémoire", assisterons-nous à un plus grand nombre de reconnaissances officielles d'actes violents commis par des collectivités? Entendrons-nous encore plus d'aveux de culpabilité et de demandes de pardon? Serons-nous plus souvent témoins de rituels ou de cérémonies de pardon? À la vue des exemples récents, il semble bien qu'une nouvelle conscience sociale du passé est en train de naître. Des sociétés et des communautés se sentent de plus en plus prêtes à accorder des pardons collectifs.

Butler voit dans ce mouvement de la mémoire plus qu'une guérison collective: «Il y a là peut-être, écrit-il, un mythe nouveau pour le futur de l'humanité, car l'enracinement dans le passé peut ouvrir un avenir prophétique». Le même auteur ira jusqu'à proposer l'institution d'une journée mondiale de la réconciliation ("a Day of Atonement") qui serait animée, non par des chefs religieux ou politiques, mais bien par des poètes: «Les poètes peuvent écrire les nouvelles liturgies de réconciliation, car la Poésie est proche de la Mémoire. Et la Mémoire est non seulement source de souffrances, mais aussi source de guérison» 50 . Dans la même ligne de pensée, Abel fait la constatation suivante: «le pardon a cette vertu quasi poétique de faire que ce monde-ci soit bien présent, mais de faire qu'il ne soit pas fini, qu'il soit susceptible d'autre chose que de se répéter» 51 .

51 O. ABEL, «Ce que le pardon vient faire dans l'histoire », Esprit 193 (1993) 62. 